\title{
The Effect of Poor Social Support on Depression among HIV/AIDS Patients in Ethiopia: A Systematic Review and Meta-Analysis
}

\author{
Adisu Birhanu Weldesenbet $\mathbb{D}^{1},{ }^{1}$ Sewnet Adem Kebede $\mathbb{D}^{2},{ }^{2}$ and Biruk Shalmeno Tusa $\mathbb{D}^{1}$ \\ ${ }^{1}$ Department of Epidemiology and Biostatistics, College of Health and Medical Sciences, Haramaya University, Haramaya, Ethiopia \\ ${ }^{2}$ Department of Epidemiology and Biostatistics, Institute of Public Health, College of Medicine and Health Sciences, \\ University of Gondar, Gondar, Ethiopia
}

Correspondence should be addressed to Adisu Birhanu Weldesenbet; adisuebi2009@gmail.com

Received 12 October 2020; Revised 23 November 2020; Accepted 28 November 2020; Published 9 December 2020

Academic Editor: Marcin Rzeszutek

Copyright (C) 2020 Adisu Birhanu Weldesenbet et al. This is an open access article distributed under the Creative Commons Attribution License, which permits unrestricted use, distribution, and reproduction in any medium, provided the original work is properly cited.

\begin{abstract}
Background. Low- and middle-income countries of which Ethiopia is one bears the high burden of depression among human immune deficiency virus and acquired immune deficiency syndrome (HIV/AIDS) patients. Several factors have been identified as being associated with increased depression among HIV/AIDS patients including poor social support. However, studies examining the effect of poor social support on depression among HIV/AIDS patients in Ethiopia have had inconsistent findings. This systematic review and meta-analysis is therefore aimed at estimating the pooled effect of poor social support on depression among HIV/AIDS patients in Ethiopia. Methods. All relevant articles published prior to July 1, 2020, were retrieved from scientific databases: PubMed, Scopus, and Google Scholar systematically. The identified studies reporting the association of depression and poor social support among HIV patients in Ethiopia were included. $I^{2}$ tests were used to assess the heterogeneity of the studies. Subgroup analysis was done based on tools to determine how pooled estimates of depression vary across tools. The pooled estimate of association between poor social support and depression was reported. Results. The aggregated metaanalysis revealed a higher odds of depression among patients with poor social support than those who had strong social support (OR: $2.31,95 \%$ CI: 1.69, 2.93). The pooled prevalence of depression among HIV/AIDS patients in Ethiopia was 38.93\% (95\%: CI: 32.01, 45.84); $\left(I^{2}=94.44 \%, p \leq 0.001\right)$. The subgroup analysis was performed based on tools, and the result showed that the highest pooled prevalence $(44.42 \%)$ was among primary studies that used the Hospital Anxiety and Depression Scale (HADS) tool. Conclusions. Human immune deficiency virus and acquired immune deficiency syndrome (HIV/AIDS) patients with poor social support were more likely to develop depression. The pooled prevalence of depression among HIV/AIDS patient was high in Ethiopia. The highest prevalence of depression was observed among studies that used HADS to screen depression. Therefore, we recommend integration of mental health and psychosocial support services into the HIV/AIDS care. Prevention of HIV/AIDS-related stigma for people with HIV/AIDS is also needed to reduce the impact of poor social support.
\end{abstract}

\section{Background}

Globally, 36.9 million people are living with human immune deficiency virus and acquired immune deficiency syndrome (HIV/AIDS) and more than half of them are in Africa. The greatest burden of the disease is concentrated in developing countries. Mental health is highly intertwined with communicable diseases such as HIV. In Ethiopia, reports indicate that the overall prevalence of HIV/AIDS is $1.1 \%$ among all ages and 2.1\% among adults. Mental disorders like depression put people at a higher risk for contracting HIV, and among those living with HIV, its associated stigmatization can lead to poor mental health outcomes [1-3].

Depression is a common mental disorder that presents with depressed mood, loss of interest or pleasure, decreased energy, feelings of guilt or low self-worth, disturbed sleep or appetite, and poor concentration. Approximately, 350 million people are currently living with depression. It is the 
fourth leading cause of disability worldwide. Its lifetime prevalence was one in five women and one in ten men $[4,5]$.

Depression is the most common mental disorder among HIV/AIDS patients with prevalence rates of about $60 \%$. Studies found that people living with HIV had twice the risk for depression than those who were at risk for HIV. Depression and HIV/AIDS are estimated to be the world's two leading causes of disability by 2030 [6, 7]. Low- and middle-income countries bear high burden of depression among HIV/AIDS patients. The prevalence of depression among people living with HIV (PLWHIV) in sub-Saharan Africa was 9 to $32 \%$ [8]. In Ethiopia, it ranges from 7.3 to $73.3 \%[9,10]$.

Mental health problems are associated with increased risk of HIV infection and interfere with their treatment, and, conversely, some mental disorders occur as a direct result of HIV infection. Depression affects a person's ability to follow treatment for HIV/AIDS. It is associated with poor adherence to antiretroviral therapy (ART) leading to immunological failure and may independently increase HIV progression [11-13].

Depression influences health-seeking behavior and outcome of HIV/AIDS treatment and increases progress and burden of disease and the risk of mortality and morbidity for HIV patients. The symptoms of depressive disorders decrease adherence to HIV ART leading to the drugresistant virus, and untreated depressive disorders decrease immune status [14-16].

Decreased social support within the context of HIV/AIDS is related to increased depression because of various factors such as educational disability and food insecurity. Low social support could result in poor adherence to medication, and as a result, poor adherence leads to immune suppression which finally leads to depression. Poor social support may lead to social isolation, which can be responsible for depression. Social isolation by HIV patients itself reduces social support that can result in a negative impact on their physical and mental well-being. This is also supported by the fact that these patients might prefer to avoid seeking help from others, and in addition, social stigma towards them could increase their isolation and loneliness [17-19].

In order to overcome the negative impact of depression among HIV/AIDS patients, evidence on association of social support with depression is of paramount importance. While the relationship between poor social support and depression is fairly well-established in high-income settings, fewer studies have investigated the association in low-resource setting like Ethiopia. Studies that have examined the association between poor social support and depression among PLWHA in Ethiopia in particular have presented inconsistent results, with some finding strong positive associations $[20,21]$ and others finding negative associations [22].

Therefore, this systematic review and meta-analysis is aimed at estimating the pooled effects of poor social support on depression among HIV/AIDS patients in Ethiopia. This finding will help decision makers and other stakeholders working on mental health to reduce the magnitude of depression and the associated disability among HIV/AIDS patients by implementing effective interventions.

\section{Methods}

2.1. The Protocol and Registration. We conducted this systematic review and meta-analysis based on the Preferred Reporting Items for Systematic Review and Meta-Analysis (PRISMA) statement guideline [23]. The protocol for this review was registered on International Prospective Register of Systematic Reviews (PROSPERO) with reference number CRD42020201157.

2.2. Search Strategies. Comprehensive search strategy was made on depressive symptoms and associated factors among HIV/AIDS patients to identify all relevant studies. A systematic literature search for the relevant papers was made in PubMed, ScienceDirect, and Google Scholar. The search was restricted to papers published prior to August 1, 2020, in Ethiopia and published in English. Population, Intervention, Comparison, and Outcome (PICO) format was used to search the relevant studies. "Social support", "Depression", Depressive symptoms, "Effect”, "HIV”, “AIDS”, and "Ethiopia" were combination of relevant keywords used.

\subsection{Eligibility Criteria}

2.3.1. Inclusion Criteria. All observational studies conducted on the prevalence of depression; studies that assessed the association of social support with depression in Ethiopia; studies published and accessible before August 1, 2020; articles written in English; and citations with abstract and/or full text were eligible for current systematic review and metaanalysis.

2.3.2. Exclusion Criteria. Articles which were not fully accessed because of the inability to assess the quality of articles in the absence of full text, duplicate reports, systematic reviews and meta-analyses, qualitative studies, and inconsistent outcome measures were excluded from the review.

2.4. Study Selection. The selection of studies from electronic databases was made based on titles and abstracts, and in cases when a definite decision could not be made based on the title and/or abstract alone, the full paper was obtained for detailed assessment of the inclusion criteria. Two authors (BST, SAK) screened and evaluated studies independently. The other author (ABW) independently evaluated the quality of the studies against the checklist, and if any discrepancy arises in decision process, it was resolved through discussion or through asking a third reviewer if consensus could not be reached. Then, the full text of the studies were further evaluated based on objectives, methods, and study populations.

2.5. Outcome Measurement. The outcome variable of interest was depression and defined as presence of depressed mood, loss of interest or pleasure, decreased energy, feelings of guilt or low self-worth, disturbed sleep or appetite, and poor concentration. In the included studies, social support is measured using the Oslo 3-item social support scale with individuals who were scored less than 9 regarded as having poor social support, adolescent social support rating scale with those scoring 1-2.9 considered having poor social 
support, and Social Support Questionnaire-6 (SSQ-6) with patients who scored below mean having low social support.

Perceived HIV stigma was measured using an 11- and 12item perceived HIV stigma scale consisting of questions about disclosures, and patients who scored greater than or equal to mean were classified as internally stigmatized. We determine the association between depression and poor social support in the form of the odds ratio.

2.6. Data Extraction Process. A data extraction template was used to extract necessary data from the articles with format containing the title of the study, author's name, study designs, year of publication, sample size, study population, and effect size by two independent groups of reviewers. Data from selected articles were extracted using a data extraction template and presented through Microsoft Word.

2.7. Quality Appraisal. The Joanna Briggs Institute (JBI) quality appraisal criteria established for analytical crosssectional studies were used. The quality of the findings of the included articles was critically evaluated using the quality assessment tool for observational studies [24]. The two groups of authors (SAK and BST) and ABW independently evaluated the quality of the studies. The reviewers compared their quality appraisal scores and resolved any discrepancy before calculating the final appraisal score. Articles with an appraisal score of $\geq 6$ out of 10 scales were considered high quality and were considered eligible.

2.8. Statistical Analysis. Standard error of proportion for all included studies was computed using the binomial distribution formula. Heterogeneity among reported prevalence was assessed by computing $p$ values of $I^{2}$-statistics. For meta-analysis with significant heterogeneity, random-effects model was used and subgroup analysis was performed. The subgroup analysis was conducted based on tools to investigate how depression varies across different categories of tools used for outcome measurement.

Publication bias was assessed by Egger's tests at 5\% significant level. Odds ratio (OR) with 95\% CI was used to examine the association between poor social support and depression. The pooled prevalence rate of depression was expressed as a point estimate and $95 \%$ CI. The prevalence from each study was weighed by the sample size. All data manipulation and statistical analysis were performed using Stata software version 16 .

2.9. Ethical Approval. Since the review was concerned with the research articles, there was no need for ethical approval and/or additional consent from participants.

\section{Results}

3.1. Search Result. Initially, a total of 1326 articles were retrieved using scientific databases (PubMed $=1254$, Science Direct $=5$, Google Scholar $=64$ ). Additional 3 articles were also searched from Google. After removing duplications, 1262 articles were considered eligible for title and abstract evaluation. Accordingly, 1232 articles were excluded and the remaining 30 articles were considered for further full-text evaluation. After the full-text reading, 22 articles were further excluded due to differences in the outcome of interests, study objectives, and overlapping of the data. Finally, 8 papers were found as eligible to be included in this systematic review and meta-analysis (Figure 1).

3.2. Characteristics of Included Studies. The studies included in this systematic review and meta-analysis were all crosssectional. They had a total of sample of 3287 adults living with HIV/AIDs in Ethiopia and were conducted from 2016 to 2020 in different regions of the country. The study sample sizes ranged from 340 reported from a study in Southern Nations Nationalities, and People (SNNP) [25] to 507 from a study in Addis Ababa [26] participants. Of the eight studies included in the final analysis, three were conducted in Addis Ababa [21, 26, 27], two studies were from the SNNPR region $[25,28]$, two studies were conducted in Amhara regions [20, $22]$, and the remaining one study was from Oromia region [29].

The response rates of the studies ranged from 93.5\% [22] to $100 \%[21,27]$. The mean age of the patients included in this systematic review and meta-analysis ranged from 18.6 \pm 3.024 years [26] to $38 \pm 10.228$ [28]. Regarding assessment tools of depressive symptoms, five studies used the Patient Health Questionnaire-9 (PHQ-9) [22, 25, 27-29], two studies used Hospital Anxiety and Depression Scale (HADS) [20, 21], and the other one study used Beck Depression Inventory-II (BDI-II) [26] (Table 1).

3.3. Meta-Analysis of Depression in Ethiopia. The prevalence of depression in this systematic review and meta-analysis ranged from $20 \%$ in Dessie [22] to $48.6 \%$ from studies in Hawassa and Bahir Dar [20,28]. The pooled prevalence of depression among HIV/AIDS patients in Ethiopia was 38.93\% (95\%: CI: 32.01\%, 45.84\%) (Figure 2).

3.4. Subgroup Analysis. There was a significant heterogeneity $\left(I^{2}=100 \%, p \leq 0.001\right)$ across the included studies. Therefore, we performed subgroup analysis based on the tool used for outcome measurement to estimate the pooled prevalence of depression in Ethiopia. The pooled prevalence of depression was $44.42 \%$ (95\% CI $37.65 \%, 52.15 \%)$ among two studies that used HADS and $37.43 \%$ (95\% CI $28.74 \%, 48.14 \%$ ) for five studies that used PHQ-9. One study indicated that the prevalence of depression among HIV/AIDS patients was $35.50 \%$ (95\% CI: 35.46, 35.54) using BDI-II (Figure 3).

3.5. The Impact of Perceived Stigma on Depression. The highest odds ratio (OR) for impact of poor social support on depression was 9.97 reported from a study conducted in Oromia region [29] and can be interpreted as the odds of having depressive symptoms among adults living with HIV who had poor social support were 9.97 times higher than those who had strong social support. The smallest odds ratio for poor social support (2.02) was reported from a study conducted in Addis Ababa [21] showing that the odds of depressive symptoms is 2.02 times more likely among those with poor social support as compared to those with strong social support. 


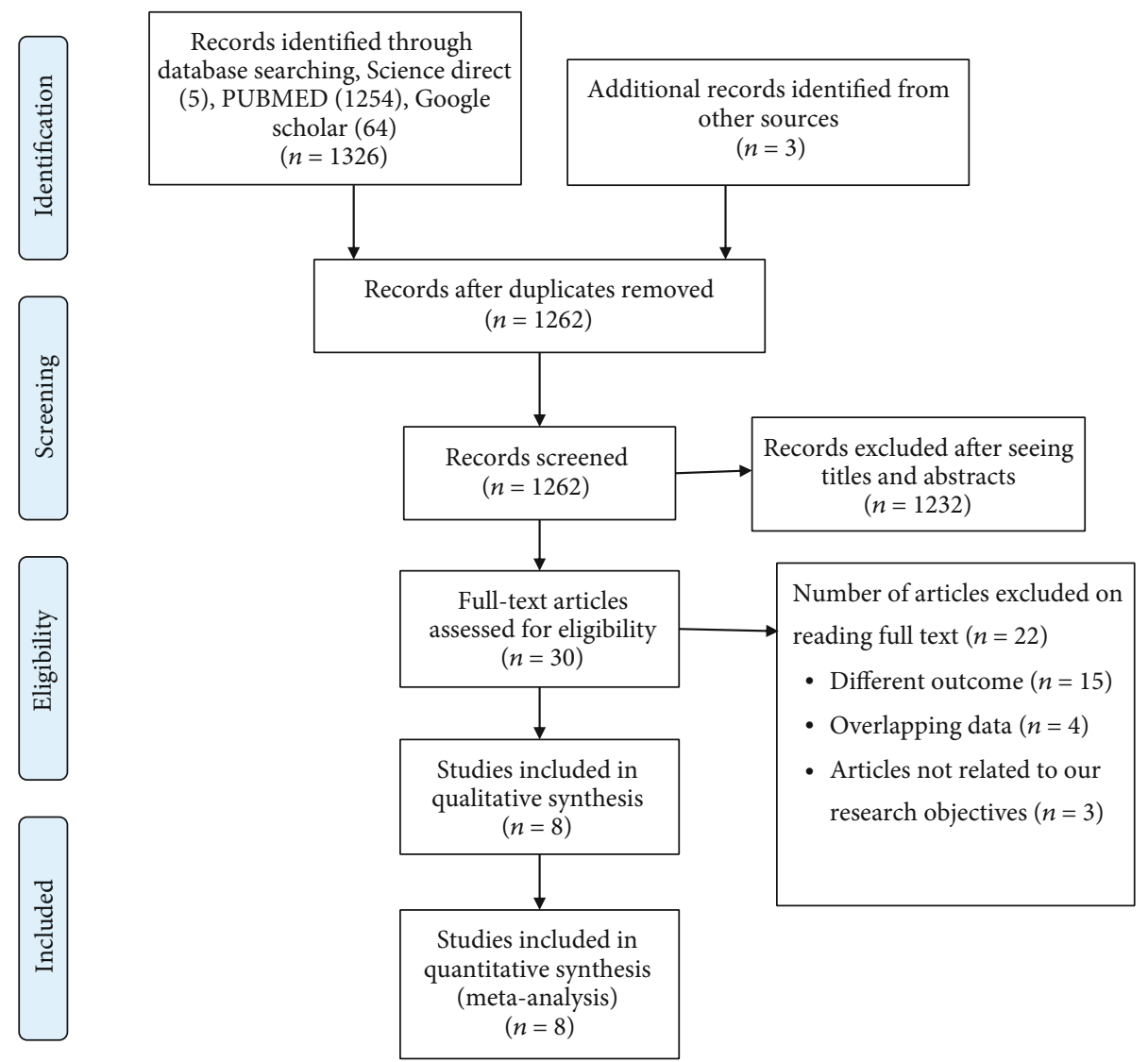

FIGURE 1: PRISMA flow chart showing the selection of primary studies.

TABlE 1: Descriptive characteristics of 8 studies included in the systematic review and meta-analysis of effect of poor social support on depression among HIV patients in Ethiopia, 2020.

\begin{tabular}{|c|c|c|c|c|c|c|c|c|}
\hline Authors (reference) & $\begin{array}{c}\text { Publication } \\
\text { year }\end{array}$ & $\begin{array}{c}\text { Study } \\
\text { area/region }\end{array}$ & Study design & Tools & $\begin{array}{l}\text { Response } \\
\text { rate }\end{array}$ & $\begin{array}{l}\text { Mean age } \\
( \pm \mathrm{SD})\end{array}$ & $\begin{array}{l}\text { Sample } \\
\text { size }\end{array}$ & Prevalence \\
\hline Duko et al. [28] & 2018 & SNNP & $\begin{array}{c}\text { Cross- } \\
\text { sectional }\end{array}$ & $\begin{array}{c}\text { PHQ- } \\
9\end{array}$ & $96 \%$ & $38 \pm 10.228$ & 417 & $48.6 \%$ \\
\hline Abadiga [29] & 2019 & Oromia & $\begin{array}{l}\text { Cross- } \\
\text { sectional }\end{array}$ & $\begin{array}{l}\text { PHQ- } \\
9\end{array}$ & $97 \%$ & $25.6 \pm 9.45$ & 404 & $41.7 \%$ \\
\hline Tesfaw et al. [21] & 2016 & Addis Ababa & $\begin{array}{l}\text { Cross- } \\
\text { sectional }\end{array}$ & HADS & $100 \%$ & $37.44 \pm 10.07$ & 417 & $41.2 \%$ \\
\hline Tareke et al. [20] & 2018 & Amhara & $\begin{array}{l}\text { Cross- } \\
\text { sectional }\end{array}$ & HADS & $98 \%$ & $36.9+10.5$ & 415 & $48.6 \%$ \\
\hline Abebe et al. [26] & 2019 & Addis Ababa & $\begin{array}{l}\text { Cross- } \\
\text { sectional }\end{array}$ & BDI-II & $94 \%$ & $18.6 \pm 3.024$ & 507 & $35.50 \%$ \\
\hline $\begin{array}{l}\text { Getalem and Emnet } \\
\text { [27] }\end{array}$ & 2016 & Addis Ababa & $\begin{array}{l}\text { Cross- } \\
\text { sectional }\end{array}$ & $\begin{array}{l}\text { PHQ- } \\
9\end{array}$ & $100 \%$ & 38 & 384 & $44.40 \%$ \\
\hline Seid et al. [22] & 2020 & Amhara & $\begin{array}{l}\text { Cross- } \\
\text { sectional }\end{array}$ & $\begin{array}{l}\text { PHQ- } \\
9\end{array}$ & $93.5 \%$ & $38(\mathrm{IQR}=10)$ & 403 & $20 \%$ \\
\hline Workye et al. [25] & 2018 & SNNP & $\begin{array}{l}\text { Cross- } \\
\text { sectional }\end{array}$ & $\begin{array}{c}\text { PHQ- } \\
9\end{array}$ & $96.5 \%$ & $37.6(R=50)$ & 340 & $37.50 \%$ \\
\hline
\end{tabular}

SD: standard deviation; SNNP: Southern Nation, Nationalities, and People; PHQ-9: Patient Health Questionnaire-9; HADS: Hospital Anxiety and Depression Scale; BDI-II: Beck Depression Inventory-II; IQR: interquartile range; R: range. 


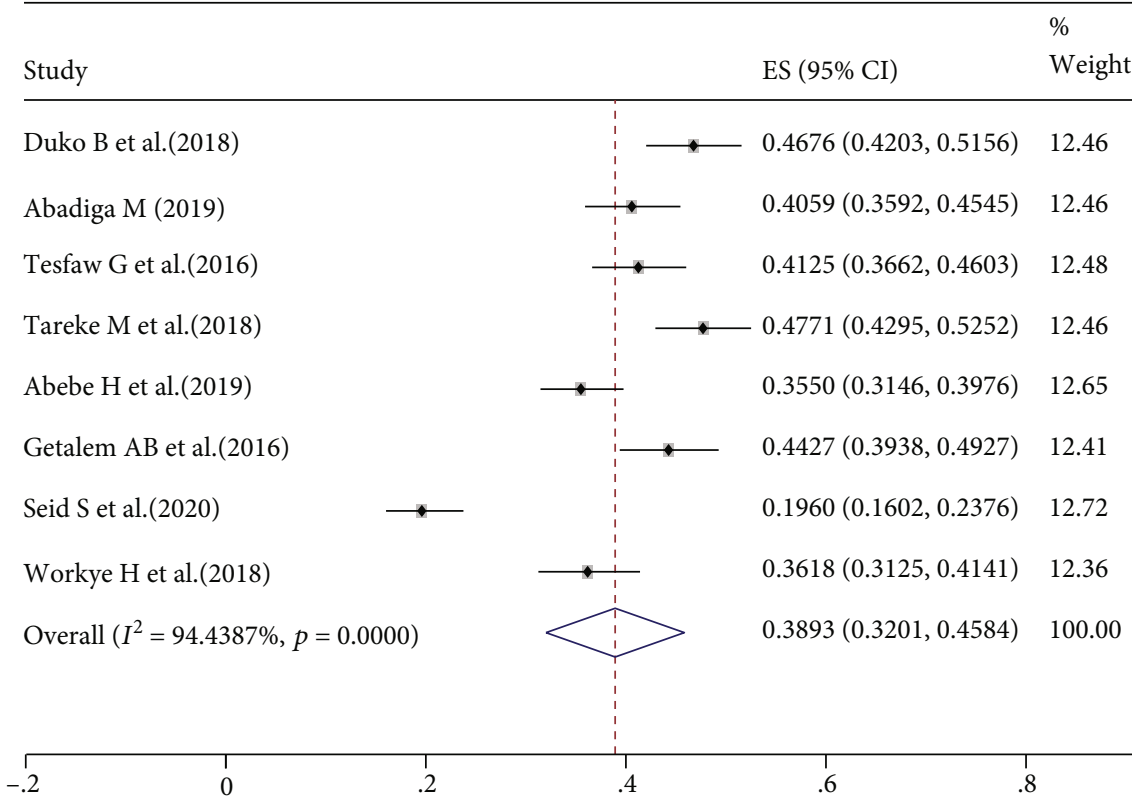

FIGURE 2: The pooled estimated prevalence of depression among HIV/AIDS patients in Ethiopia, 2020.

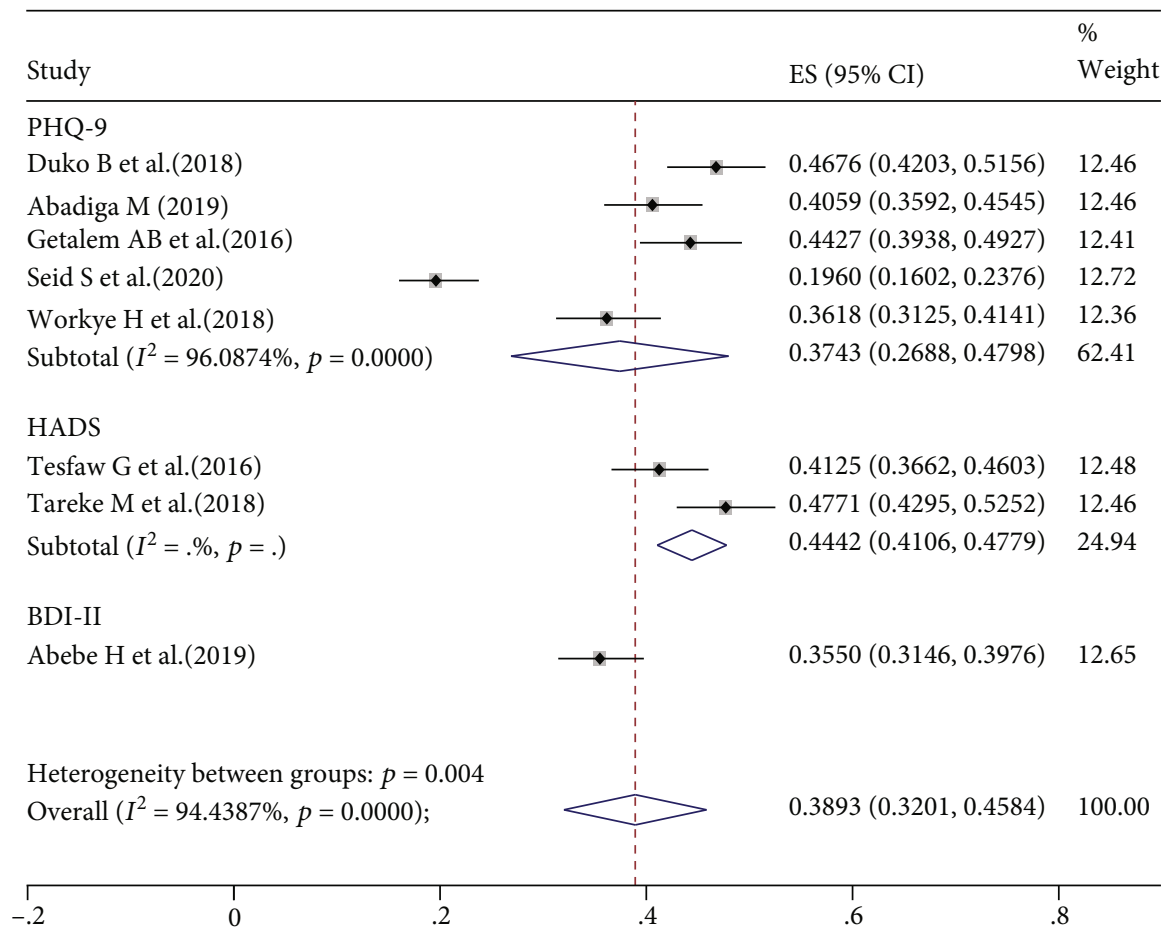

FIGURE 3: The pooled estimated prevalence of depression among HIV/AIDS patients in Ethiopia based on tools, 2020.

In seven of eight included studies [20, 21, 25-29], there was a significant association between poor social support and depression. The aggregated meta-analysis using eight studies revealed HIV/AIDS patients with poor social support had 2.31 times higher odds of developing depression as compared to those who had strong social support (OR: 2.31, 95\% CI: 1.69, 2.93) (Figure 4).
3.6. Publication Bias Results. Funnel plot and Egger's test was used to evaluate the presence of publication bias. Each dot in the funnel plot represents a single study. The $y$-axis is usually the standard error of odds ratio. Larger studies with higher power are placed towards the top whereas lower powered studies are placed towards the bottom. The $x$-axis shows the odds ratio. 


\begin{tabular}{|c|c|c|c|}
\hline \multicolumn{3}{|l|}{ Study } & $\%$ \\
\hline \multicolumn{2}{|l|}{ ID } & ES (95\% CI) & Weight \\
\hline Duko B et al.(2018) & $\leftarrow$ & $2.53(1.70,4.51)$ & 19.60 \\
\hline Abadiga M (2019) & & $\rightarrow 9.97(3.57,27.86)$ & 0.26 \\
\hline Tesfaw G et al.(2016) & $\leftarrow$ & $2.02(1.25,3.27)$ & 37.93 \\
\hline Tareke M et al.(2018) & $\leftarrow$ & $2.04(1.06,3.93)$ & 18.79 \\
\hline Abebe $\mathrm{H}$ et al.(2019) & $\leftrightarrow$ & $2.74(1.42,5.27)$ & 10.44 \\
\hline Getalem AB et al.(2016) & $\rightarrow$ & $3.36(1.71,6.61)$ & 6.45 \\
\hline Workye $\mathrm{H}$ et al.(2018) & + & $4.00(1.72,9.27)$ & 2.71 \\
\hline Seid et al. (2020) & 4 & $0.67(0.07,6.43)$ & 3.83 \\
\hline Overall $\left(I^{2}=0.0 \%, p=0.689\right)$ & A & $2.31(1.69,2.93)$ & 100.00 \\
\hline-27.9 & 0 & 27.9 & \\
\hline
\end{tabular}

FIGURE 4: Forest plot of pooled OR between poor social support and depression among HIV/AIDS patients in Ethiopia.

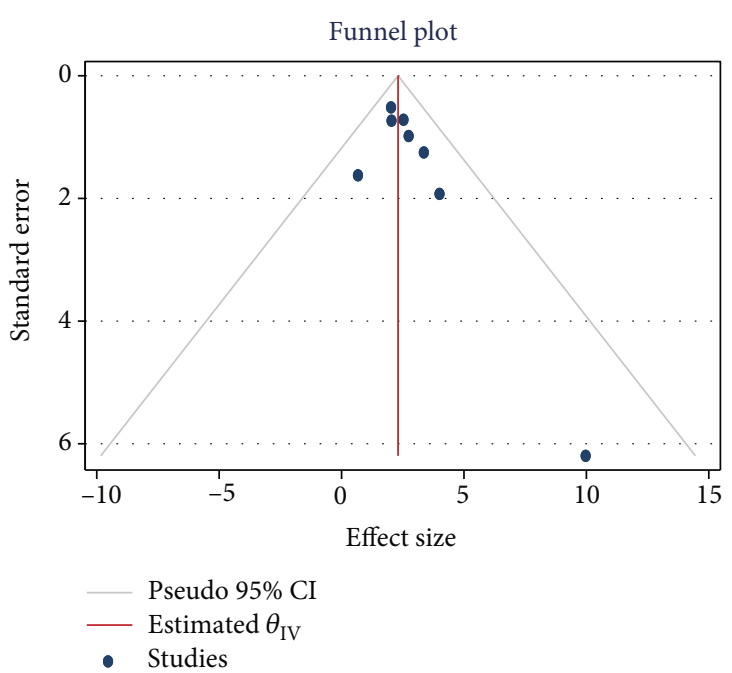

Figure 5: A funnel plot for assessing publication bias.

For the current review as depicted in Figure 5, the plot is symmetric indicating there is no evidence of publication bias or small study effect in our study ( $p$ value from Egger's test $=0.065$ ). However, in this interpretation, it should be put into consideration that the funnel plot and Egger's test are less reliable when the number of studies is less than 10 .

\section{Discussion}

This systemic review and meta-analysis attempted to estimate the pooled effect of poor social support on depression among HIV/AIDS patients in Ethiopia. The analysis revealed that poor social support had a statistically significant effect on depression among HIV/AIDS patients. The odds of hav- ing depression among adults living with HIV who had poor social support were $31 \%$ higher than those who had strong social support. The positive relationship between poor social support and depression in the current study is consistent with a finding from a systematic review in Africa [30]. The reason might be due to the fact that patients who did not share their problems with other people had stress and social isolation by HIV patients itself reduces social support that can result in a negative impact on their physical and mental well-being. The fact that these patients might prefer to avoid seeking help from others and in addition, social stigma towards them could increase their isolation and loneliness might also contribute to positive relationship between poor social support and depression $[18,19]$.

The pooled prevalence of depression among HIV/AIDS patients in the current study was $38.93 \%$. This finding is in line with the prevalence of depression among HIV/AIDS reported from low- and middle-income countries (12 to 78\%) [31]. The current finding was higher than the study in sub-Saharan Africa (9 to 32\%), and the possible reason might be due to the difference in sample size, methodology, and tools used to assess depression.

We conducted subgroup analysis based on tools, and the highest prevalence was observed among primary studies that used HADS (44.42\%), and it was $37.43 \%$ among primary studies that used the PHQ-9 tool. The disparity might be due to the difference in the number of primary studies. Only two primary studies used HADS as a tool to assess depression whereas five studies used PHQ-9, and this may increase precision of the pooled estimate for depression.

The strength of this review is it was conducted with rigorous adherence to the PRISMA checklist which improves its quality for the readers. The main limitation of this metaanalysis is it may be lacking national representativeness since 
primary studies were found only from four administrative regions, namely, Addis Ababa, Amhara region, Oromia region, and SNNP region; this could bias the estimated prevalence of depression for the entire Ethiopian context.

\section{Conclusions}

The pooled prevalence of depression among HIV/AIDS patient was high in Ethiopia. The highest prevalence of depression was observed among studies that used HADS. Human immune deficiency and acquired immune deficiency syndrome patients with poor social support were more likely to develop depression. Therefore, we recommend the integration of mental health and psychosocial support services into the HIV/AIDS care. The prevention of HIV/AIDSrelated stigma for people with HIV/AIDS is also needed to reduce the impact of poor social support.

\section{Abbreviations}

$\begin{array}{ll}\text { AIDS: } & \text { Acquired immune deficiency syndrome } \\ \text { ART: } & \text { Antiretroviral therapy } \\ \text { BDI-II: } & \text { Beck Depression Inventory-II } \\ \text { CI: } & \text { Confidence interval } \\ \text { HADS: } & \text { Hospital Anxiety and Depression Scale } \\ \text { HIV: } & \text { Human immune deficiency virus } \\ \text { JBI: } & \text { Joanna Briggs Institute } \\ \text { OR: } & \text { Odd ratios } \\ \text { PHQ-9: } & \text { Patient Health Questionnaire-9 } \\ \text { PICO: } & \text { Population intervention comparison and } \\ & \text { outcome } \\ \text { PLWHA: } & \text { People living with HIV/AIDS } \\ \text { PRISMA: } & \text { Preferred Reporting Items for Systematic } \\ & \text { Review and Meta-Analysis } \\ \text { PROSPERO: } & \text { Prospective Register of Systematic Reviews } \\ \text { SSQ-6: } & \text { Social Support Questionnaire-6 } \\ \text { SNNP: } & \text { Southern Nation, Nationalities, and People. }\end{array}$

\section{Data Availability}

All necessary information were included with in the manuscript.

\section{Consent}

Not applicable.

\section{Conflicts of Interest}

The authors declare that they have no competing interests, financial, or otherwise.

\section{Authors' Contributions}

ABW had a primary role in conceptualization, data review, data extraction, data analysis, and writing and editing of this manuscript. BST and SAK had a role in data review, data extraction, and writing and editing of this manuscript. All the authors approved the final version of the manuscript.

\section{References}

[1] M. Prince, V. Patel, S. Saxena et al., "No health without mental health," The lancet, vol. 370, no. 9590, pp. 859-877, 2007.

[2] WHO, "Mental health and development: targeting people with mental health conditions as a vulnerable group," Mental health and development: targeting people with mental health conditions as a vulnerable group, p. 74, 2010.

[3] WHO, World Health Organization data and statistics on HIV, 2017.

[4] WHO, World suicide prevention day: prepared by World Health Organization, 2012.

[5] J. S. Benjamin, VAT, Getachew. Behavioral Sciences/Clinical Psychiatry, Lippincott Williams \& Wilkins, Kaplan \& Sadock's Synopsis of Psychiatry, New York University, School of Medicine, New York, 10th edition, 2007.

[6] J. A. Ciesla and J. E. Roberts, "Meta-analysis of the relationship between HIV infection and risk for depressive disorders," American Journal of Psychiatry, vol. 158, no. 5, pp. 725-730, 2001.

[7] C. D. Mathers and D. Loncar, "Projections of global mortality and burden of disease from 2002 to 2030," PLoS medicine, vol. 3, no. 11, article e442, 2006.

[8] C. Bernard, F. Dabis, and N. de Rekeneire, "Prevalence and factors associated with depression in people living with HIV in sub-Saharan Africa: a systematic review and meta-analysis," PloS one, vol. 12, no. 8, article e0181960, 2017.

[9] W. M. Bezabhe, L. Chalmers, L. R. Bereznicki, P. Gee, and G. M. Peterson, "Antiretroviral adherence and treatment outcomes among adult Ethiopian patients," AIDS care, vol. 28, no. 8, pp. 1018-1022, 2016.

[10] B. Tolasa, B. Dereje, T. Temamen, and T. Belachew, "Food insecurity and associated factors among people living with HIV attending ART Clinic in Fitche zonal hospital, Ethopia," Journal of Pharmacy and Alternative Medicine, vol. 8, no. 3, pp. 8-17, 2015.

[11] NIMH, Depression and HIV/AIDS, National Institute of Mental Health, United States of America (USA), 2011.

[12] WHO, HIVIAIDS and mental health report by the Secretariat, 2008.

[13] N. N. Deshmukh, A. M. Borkar, and J. S. Deshmukh, "Depression and its associated factors among people living with HIV/AIDS: can it affect their quality of life?," Journal of family medicine and primary care, vol. 6, no. 3, p. 549, 2017.

[14] A. Au, C. Cheng, I. Chan, P. Leung, P. Li, and R. K. Heaton, "Subjective memory complaints, mood, and memory deficits among HIV/AIDS patients in Hong Kong," Journal of Clinical and Experimental Neuropsychology, vol. 30, no. 3, pp. 338-348, 2008.

[15] S. H. Tesfaye and G. T. Bune, "Generalized psychological distress among HIV-infected patients enrolled in antiretroviral treatment in Dilla University Hospital, Gedeo zone, Ethopia," Global health action, vol. 7, no. 1, article 23882, 2014.

[16] C. S. Brennan, "Untreated depressive disorders have big impact on HIV," HIV clinician, vol. 22, no. 3, pp. 6-9, 2010.

[17] J. W. Kamau, W. Kuria, M. Mathai, L. Atwoli, and R. Kangethe, "Psychiatric morbidity among HIV-infected children and adolescents in a resource-poor Kenyan urban community," AIDS care, vol. 24, no. 7, pp. 836-842, 2012.

[18] R. Nüesch, A. Gayet-Ageron, P. Chetchotisakd et al., "The impact of combination antiretroviral therapy and its 
interruption on anxiety, stress, depression and quality of life in Thai patients," The open AIDS journal, vol. 3, p. 38, 2009.

[19] H. Berhe and A. Bayray, "Prevalence of depression and associated factors among people living with HIV/AIDS in tigray, North Ethiopia: a cross sectional hospital based study," International Journal of Pharmaceutical Sciences and Research, vol. 4, no. 2, p. 765, 2013.

[20] M. Tareke, F. Addisu, and A. Abate, "Depression among patients attending antiretroviral treatment program in public health facilities in Bahir Dar City, Ethiopia," Journal of affective disorders, vol. 232, pp. 370-374, 2018.

[21] G. Tesfaw, G. Ayano, T. Awoke et al., "Prevalence and correlates of depression and anxiety among patients with HIV onfollow up at Alert Hospital, Addis Ababa, Ethopia," BMC psychiatry, vol. 16, no. 1, p. 368, 2016.

[22] S. Seid, O. Abdu, M. Mitiku, and K. S. Tamirat, "Prevalence of depression and associated factors among HIV/AIDS patients attending antiretroviral therapy clinic at Dessie referral hospital, South Wollo, Ethiopia," International Journal of Mental Health Systems, vol. 14, no. 1, pp. 1-8, 2020.

[23] D. Moher, L. Shamseer, M. Clarke et al., "Preferred reporting items for systematic review and meta-analysis protocols (PRISMA-P) 2015 statement," Systematic reviews, vol. 4, no. 1, p. $1,2015$.

[24] K. Porritt, J. Gomersall, and C. Lockwood, "JBI's systematic reviews: study selection and critical appraisal," AJN The American Journal of Nursing, vol. 114, no. 6, pp. 47-52, 2014.

[25] H. W. Agazhu, B. W. Giru, and T. H. Wurjine, "Prevalence and associated factors of depression among HIV/AIDS patients attending anti-retroviral therapy clinics at Gurage zone selected government hospitals, Southwest, South Nations, Nationalities and Peoples' Region, Ethiopia," International Journal of Scientific \& Engineering Research, vol. 9, 2018.

[26] H. Abebe, S. Shumet, Z. Nassir, M. Agidew, and D. Abebaw, "Prevalence of depressive symptoms and associated factors among HIV-positive youth attending ART follow-up in Addis Ababa, Ethiopia," AIDS Research and Treatment, vol. 2019, 7 pages, 2019.

[27] A. Getalem and A. F. Emnet, "Prevalence and associated factors of depression among HIV patients taking antiretroviral therapy at Zewditu Memorial Hospital, Addis Ababa, Ethopia," Journal of Scientific Research and Studies, vol. 3, no. 4, pp. 81-86, 2016.

[28] B. Duko, E. Geja, M. Zewude, and S. Mekonen, "Prevalence and associated factors of depression among patients with HIV/AIDS in Hawassa, Ethiopia, cross-sectional study," Annals of general psychiatry, vol. 17, no. 1, p. 45, 2018.

[29] M. Abadiga, "Depression and its associated factors among HIV/AIDS patients attending ART clinics at Gimbi General hospital, West Ethiopia," BMC Research Notes, vol. 12, no. 1, p. 527, 2018.

[30] R. O. Shittu, M. K. Alabi, L. O. Odeigah et al., "Suicidal ideation among depressed people living with HIV/AIDS in Nigeria, West Africa," Open Journal of Medical Psychology, vol. 3, no. 3, pp. 262-270, 2014.

[31] O. A. Uthman, J. F. Magidson, S. A. Safren, and J. B. Nachega, "Depression and adherence to antiretroviral therapy in low-, middle-and high-income countries: a systematic review and meta-analysis," Current Hiv/aids Reports, vol. 11, no. 3, pp. 291-307, 2014. 